\title{
Correction to: Bartonella and Rickettsia Infections in Haematophagous Spinturnix myoti Mites (Acari: Mesostigmata) and their Bat Host, Myotis myotis (Yangochiroptera: Vespertilionidae), from Poland
}

\author{
Agnieszka Szubert-Kruszyńska ${ }^{1}$ - Joanna Stańczak ${ }^{2} \cdot$ Stella Cieniuch $^{2} \cdot$ Edyta Podsiadły $^{3} \cdot$ Tomasz Postawa $^{4}$. \\ Jerzy Michalik ${ }^{5}$ (D)
}

Published online: 8 September 2018

(C) Springer Science+Business Media, LLC, part of Springer Nature 2018

Correction to: Microbial Ecology

https://doi.org/10.1007/s00248-018-1246-5

The original version of this article published online (27 August 2018) unfortunately contained a mistake regarding an affiliation of Dr. Edyta Podsiadły, one of the authors.

The correct affiliation version of Dr. Edyta Podsiadły is presented below:

3 Department of Microbiology, Faculty of Medicine, University of Rzeszów, Rzeszów, Poland.

an incorrect affiliation address published in online version was:

${ }^{3}$ Department of Laboratory Diagnostics and Clinical Immunology of Developmental Age, Public Pediatric Teaching Hospital, Warszawa, Poland.

The online version of the original article can be found at https://doi.org/ $10.1007 / \mathrm{s} 00248-018-1246-5$

Jerzy Michalik

michalik@amu.edu.pl

1 Evolutionary Biology Group, Faculty of Biology, Adam Mickiewicz University, Poznan, Poland

2 Department of Tropical Parasitology, Institute of Maritime and Tropical Medicine, Medical University of Gdańsk, Gdynia, Poland

3 Department of Microbiology, Faculty of Medicine, University of Rzeszów, Rzeszów, Poland

4 Institute of Systematic and Evolution of Animals, Polish Academy of Sciences, Krakow, Poland

5 Department of Animal Morphology, Faculty of Biology, Adam Mickiewicz University, Umultowska 89, 61-614 Poznan, Poland 